\title{
Checking Relationship Consistency and Class Redundancy in a Class Diagram under Model-Driven Engineering
}

\author{
Liang Huang ${ }^{1}$, Yucong Duan ${ }^{2 *}$, Honghao $\mathrm{Gao}^{3}, \mathrm{Hui}^{2}{ }^{4}$, Caimao $\mathrm{Li}^{5}$ \\ and Zhiyang Lin $^{6}$ \\ ${ }^{1,2,4,5,6}$ College of Information Science and Technology, Hainan University, China \\ ${ }^{3}$ College of Computer Science and Technology, Zhejiang University, \\ Hangzhou, China \\ 111512460987@qq.com,2yucongduan@hotmail.com, ${ }^{3}$ gaohonghao@zju.edu.cn, \\ hitlihui1112@163.com, ${ }^{5}$ Lilcaim@126.com
}

\begin{abstract}
Model-Driven Engineering (MDE) tries to reduce the effort spent on software development by generating codes from models. People condentrate their minds on the transformation between models and models, or between models and codes. People also concentrate on checking consistency between different models such as consistency between a class diagram and a sequence diagram and consistency between a sequence diagram and a state machine diagram. Checking relationship consistency and class redundancy in a class model is still important but ignôredin recent years. This paper concentrates on relationship problems between classes in a class diagram and proposes methods of checking various relationship problems. We address the redundancy of a class's operations and attributes. We identify a large range of the problems for class diagram. Our research is based on the relationship abstraction rules.
\end{abstract}

Keywords: class diagram, retationship abstraction, circulation inconsistency, class redundancy

\section{Introduction}

A model is an abstract representation of a real system or phenomenon [11]. Models are created to meet particular purposes, for example, to present a human understandable description of some aspec of a system or to present information in a form that can be mechanically analyzed [13]. Models and modeling are essential parts of every engineering endeavors [1]. We buind models in order to get a better understanding of the structures and behaviors of our software systems [10]. Well constructed models make it easier to deliver large, complex enterprise systems on time and within budgets [7]. OMG launched the Moder Driven Architecture (MDA) as a Model-Driven Engineering (MDE) standard in 2001 9,12$]$. The promise of MDE is that the development and maintenance cost can be pecuced by working with models instead of codes [14]. In traditional software development, codes are core elements. However software system of today's are increasing more and more complexity, distribution, heterogeneity and lifespan [5]. Traditional software development methods cannot meet the changing requirement of users and cannot catch the essence of the problem area. But in MDA, models become core elements in software development and are not only used as an assistant tool.

Our research on model checking is based on the Unified Modeling Language (UML). UML is a de-facto standard for object-oriented modeling [4]. Relevant information in different UML diagrams [2] under one project should be consistent with each other. In MDE we may need to check consistencies between diagrams. 
Checking consistency between two diagrams (CMod). For a given software project $(\mathrm{P})$, stakeholders propose a requirement $(\mathrm{R})$. In order to model $\mathrm{P}$, developers use two different diagrams (D1), (D2). In D1, the information used to describe R is (INFO1). In D2, the

CMod: $\forall \mathrm{p} \in \mathrm{P}, \exists \mathrm{d} 1 \in \mathrm{D} 1 \wedge \exists \mathrm{d} 2 \in \mathrm{D} 2 ; \forall \mathrm{r} \in \mathrm{R}, \exists$ info1 $\in \mathrm{INFO} 1 \wedge \exists$ info2 $\in \mathrm{INFO} 2$; $($ DIFF $($ infol, info 2$)=$ True $) \Rightarrow(\operatorname{INCON}(\mathrm{d} 1, \mathrm{~d} 1)=$ True $)$;

information used to describe R is (INFO2). If INFO1 is different from INFO2 (DIFF), we conclude that D1 is inconsistent with D2 (INCON).

Consistency checking between models is an important part in developing models. Nuseibeh proposed that consistency checking is an activity focusing on comparing information in two or more views [8]. This implies that one important goal of view integration is to provide automatic assistance in identifying view inconsistencies [3].

Three types of inconsistency are identified:

1). Inconsistency between two class diagrams. It means the inconsistency between a low level class diagram and a high level class diagram. The high level class diagram is abstracted from the low level class diagram. For this kind of inconsistency, when we check a model using the method CMod, one of D1 and D2 is a high leyel clas diagram and the other is a low level class diagram.

2). Inconsistency between a class diagram and a sequence diagram. Mmeans the call between classes in sequence diagram doesn't match their relationship $\mathrm{in}$ class diagram. When using CMod to check that, one of D1 and D2 is a class diagram and the other is a sequence diagram.

3). Inconsistency between a state machine and a sequênce diagram. It means the states in a state machine don't match the execution sequence in a sequence diagram. For example, an object sends a message to another object ln a sequence diagram, but in a state machine diagram there is no state to describe the state change. When using CMod to check that, one of D1 and D2 is a state machine diagram and the other is a sequence diagram.

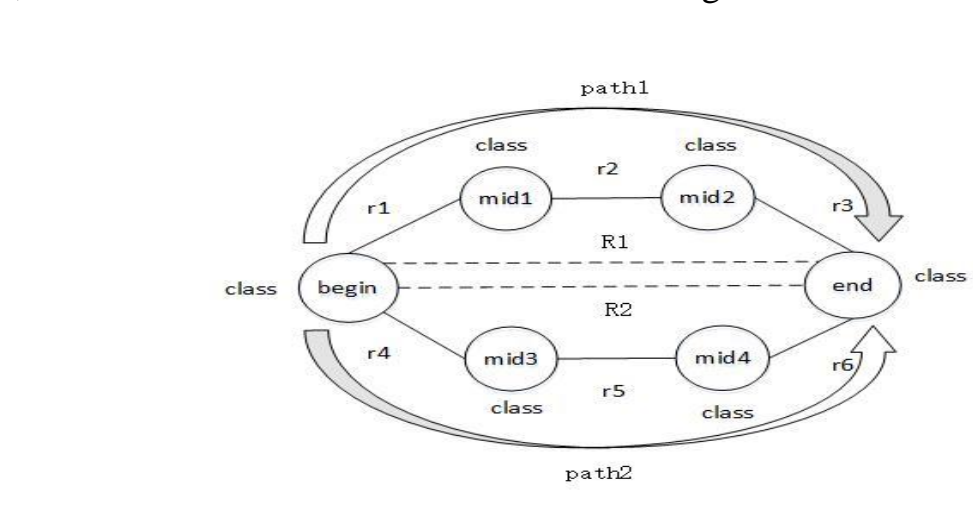

Figure 1. A Form of Circulation

In this paper, we investigate relationship problems in a single class diagram. We also investigate the redundancy of attributes and operations in a class diagram and circulation problems in a class diagram. The circulation may have problems shown in Figure 1. In Figure 1, there are two paths between class begin and class end. The two paths are path1 and path2. $\{r 1, r 2, r 3\} \in$ pathl. $r 1, r 2$ and $r 3$ are relationships. If we abstract $r 1, r 2$ and $r 3$ in pathl, we can get a direct relationship $R 1$ between class begin and class end. If we abstract $r 4, r 5$ and $r 6$ in path2, we can get a direct relationship $R 2$ between class begin and class end. If $R 1 \neq R 2$, we conclude that path 1 is inconsistent with path 2 and the circulation is not correct. 


\section{Checking Relationships}

If the size of a class diagram is small, we can check relevant problems on relationships and classes manually. The checking includes consistency checking, redundancy checking and completeness checking. However, when the software system becomes larger and more complex, we are impossible to find out all the problems manually. Using a tool to discover the problems in a diagram helps us to correct the diagram easily. We find that relationship problems mainly exist in circulations as shown in Figure 1. Figure 2 is an activity diagram indicating the activities in relationship checking. The activities contain circulation finding, relationship consistency checking, completeness checking and redundancy checking. The progress of the activity diagram is:

1). Using the depth-first search (DFS) method to find out all the circulations in the class diagram.

2). Choosing two classes for each circulation. For the two selected classes, we use relationship abstraction rules to abstract the relationships between them. We get tyo relationship results $R 1$ and $R 2$ of two classes in Figure 1, because there are two paths namely path 1 and path 2 between them.

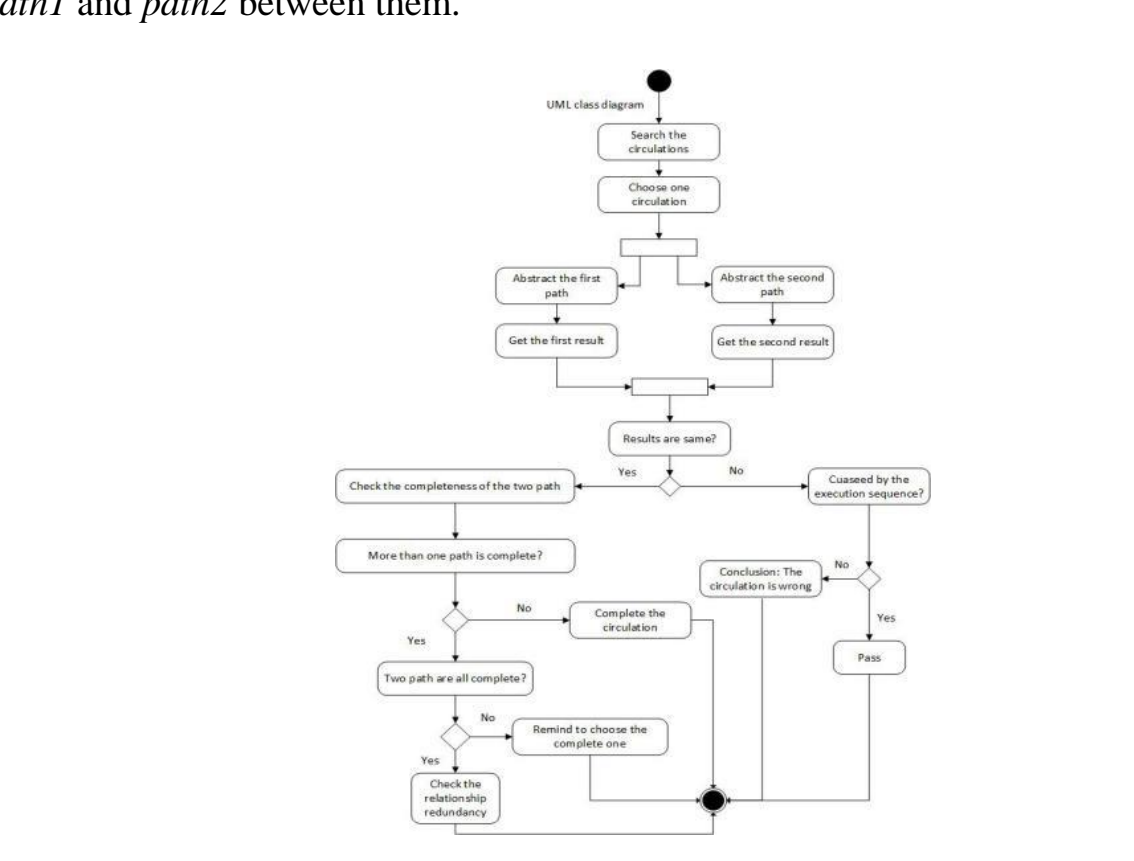

Figure 2. The Activity Diagram of Finding and Checking Circulations

3). Comparing the two relationship results. If the two results are identical, we then check the completeness of information for each path. If the information of two paths is complete, we check the relationship redundancy in the circulation. If the information of one path is complete and the other one is not complete, we choose the path in which the information is complete to satisfy the requirements of stakeholders. If both two paths are not complete, we may create a new direct relationship between the two classes.

4). Finding The reason of their difference. The first reason may be the different execution order. The second factor may be the path inconsistency. If the relationship difference is caused by the second reason, the circulation is not correct. We ignore the first reason. In the section 3, we will analyze the difference caused by the rules' execution sequences.

\subsection{Searching Circulations in a Class Diagram}

Figure 3 is a simple class diagram containing circulations. The left part of Figure 3 shows a class diagram. The right part of Figure 3 shows the relationships in the class 
diagram. We consider the class diagram as a graph. We use the DFS method to get circulations in the class diagram.

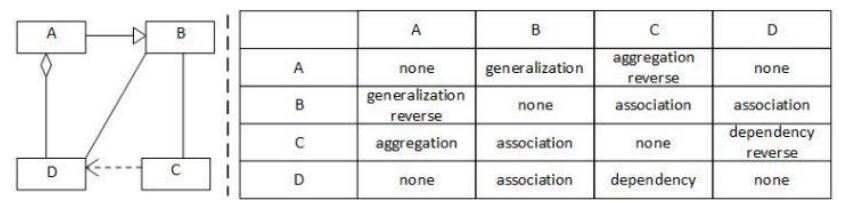

Figure 3. A Class Diagram Contains Circulations

\subsection{Abstraction and Abstraction Rules}

If we want to get the direct relationship between two classes, we need rules to abstract the paths between the two classes. Abstraction contains relationship abstraction and classifier abstraction. Classifier abstraction uses the classification and clusering method to get a high level abstraction. The package diagram in UML introduces the method of using classifier abstraction. Relationship abstraction abstracts the relationships in the class diagram. For example, relationship abstraction find the relation between two relationships and abstract the two relationships int one. The relationship abstraction uses rules to abstract the class diagram and abstracts a low level class diagram to a higher level class diagram.

1). Grammars of relationship abstraction and classifier abstraction. Grammars of

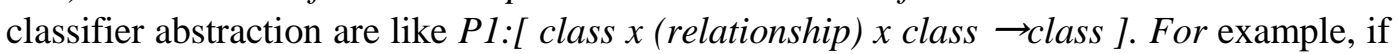
in a scene class A and class B has a relationship accor ing to grammar $P 1$, the scene can be abstracted on class A. The grammar of relationship abstraction is P2:[ (relationship) $x$ class $x$ (relationship) $\rightarrow$ relationship ]. For example, if in a scene class A has a relationship with class $\mathrm{B}$ and class $\mathrm{B}$ has a relationship with class $\mathrm{C}$ according to $P 2$, the scene indicates that class A has a relationship with class C. Figure 4 is a scene describing the relationship between class Human, Mammals and Animals. Human is a kind of mammals and a maminal is a kind of animals. The scene can be described as S1:[ Human $x$ (Generalization) XMammals (Generalization) $x$ Animals $]$. According to the classifier abstraction grammar:[ Human $x$ Generalization) $x$ Mammals $\rightarrow$ Human ], We abstract the scene $S 1$ and we get the abstracted scene which describes human and animal is $S 2:[$ Human $x$ (Generalization) Animal ]. If we use the relationship abstraction to abstract the scene, according to the relationship abstraction rule:[ (Generalization) $x$ Class $x$ (Generalization) $\rightarrow$ Generalization ], we can get an Generalization relationship. And finally we can get the abstracted scene which describes Human and Animal is S3:[ Human $x$ (Generalization) $x$ Animal ]. Relationship abstraction means that the model is abstracted through relationship abstraction rules rather than classifier abstraction rules. The relationship abstraction is used to get a higher-level abstraction of a class diagram and the relationship abstraction is used to abstract paths.

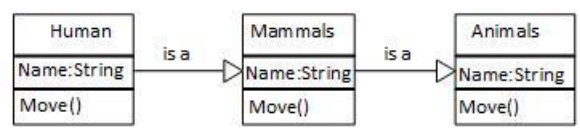

Figure 4. Animal Family Example

2). Input and output for relationship abstraction and classifier abstraction. Figure 5 shows the input and output of relationship abstraction and classifier abstraction. The upper part of Figure 5 indicates the input and output for the classifier abstraction. The 
upper part of Figure 5 matches the grammar of classifier abstraction P1:[ Class $x$ (Relationship) $x$ Class $\rightarrow$ Class ]. If we input [Class $1 \times$ (Relationship) $x$ Class2 ], the output is Class 1 . The lower part of Figure 5 indicates the input and output for relationship abstraction. The lower part of matches the grammar of relationship abstraction P2:[ (Relationship) $x$ Class $x$ (Relationship) $\rightarrow$ Relationship ]. If we input [ (Relationship1) $x$ Class $x$ (Relationship2) ], the output is Relationship1 or Relationship2. The output of a relationship abstraction depends on the relationship abstraction rules.

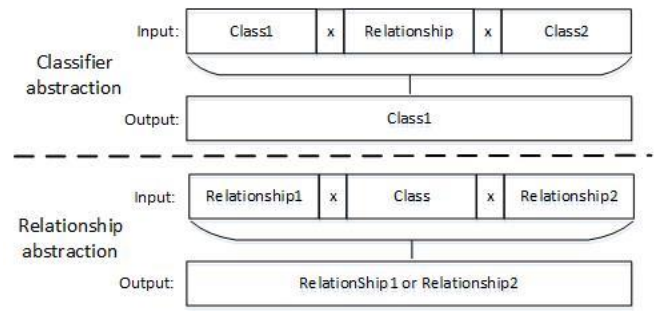

Figure 5. Input and Output for Abstraction

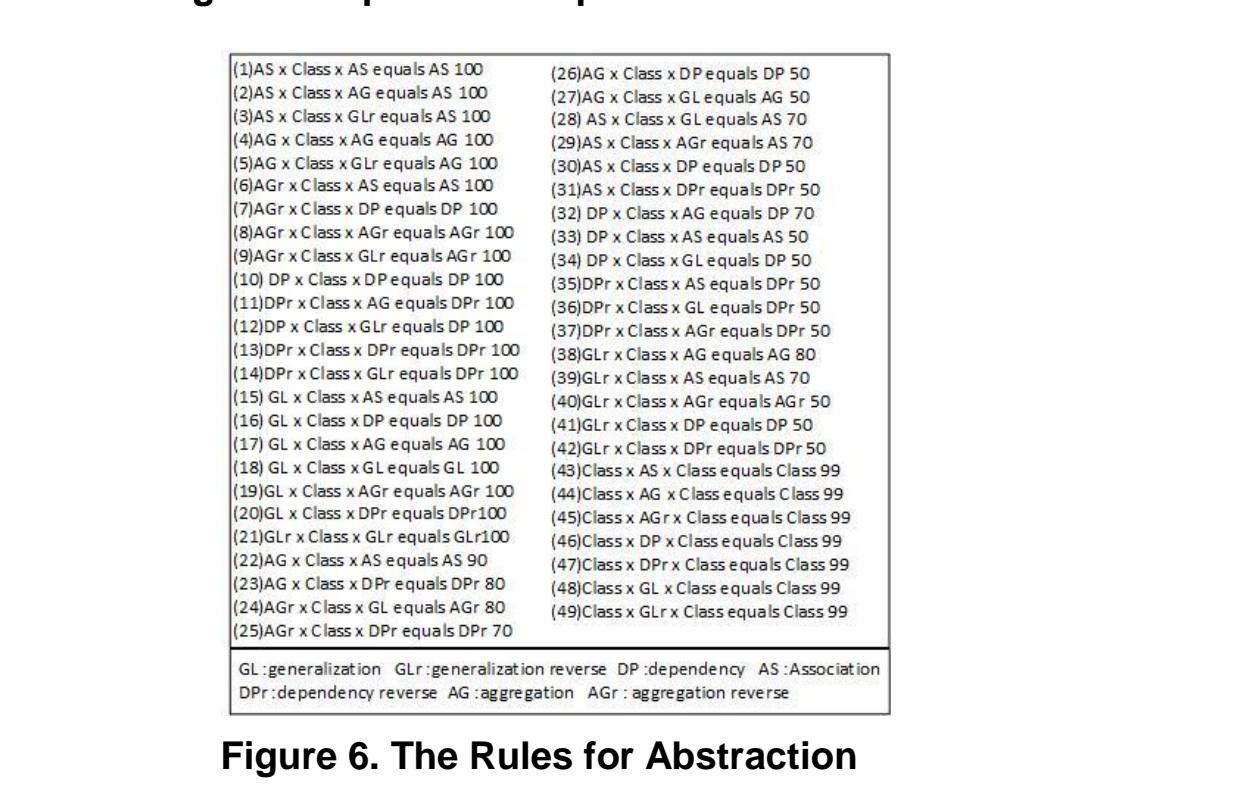

3). Abstraction rules and reliability. UML relationships include dependency, aggregation, assocration, generalization and composition. The former four kinds of relationships âe frequently used. The combination with repetition of any two relationships can reflect in an input structure. And the input structure belongs to relationsiitp abstraction input structure. If two classes are connected with a relationship, a combination of the two classes and relationship can reflect on an input structure. And this input structure belongs to the classifier abstraction input structure. Figure 6 shows relevant mies for relationship abstraction and classifier abstraction. The part before "equals" in each rule is similar to the part before " $\rightarrow$ " in grammar. The part meets the input structure. The part after "equals" is similar to the part after " $\rightarrow$ " in grammar. The part meets with the output structure. The number at the end of the rule shows the reliability of the abstraction rule. We get the final reliability after running two or more rules. For example, the reliability of rule (23) is 80 and the reliability of rule (30) is 50. The ultimate reliability is $80 * 50 / 100=40$. The lower part of Figure 6 shows the short hands for the relationships. In Figure 6, there are 42 relationship abstraction rules and 7 classifier abstraction. The rules ranging from (1) to (42) belong to relationship abstraction rules. The rules ranging from (43) to (49) belong to classifier abstraction rules. In the 
relationship abstraction rules, there are 21 rules ranging from (1) to (21) whose reliability are 100 , taking nearly half of the relationship abstraction rules.

\subsection{Problems in Circulation}

A relationship circulation in a class diagram requires that there are two paths from one class to another class. If class A wants to get the information of class B, there will be two paths for class A to choose from. Circulation often contains problems. For example, we may select the wrong path. We get two relationships between class A and class B after we abstract the two paths. If the two relationships are significantly different, the diagram contains inconsistency. Another problem is relationship redundancy in the circulation.

1). Relationship consistency:

Check relationship consistency in a circulation (CkCir). We suppose that class diagram (D) contains circulation (C). C contains two classes (CL1) and (CL2). Between CL1 and CL2, there are two paths (P1) and (P2). Using abstraction rules (Abs), we abstract P1 and get a direct relationship (R1) between CL1 and CL2. We abstract P2 and get a direct relationship (R2) between CL1 and CL2. If $R 1 \neq R 2$, we say that P1 is inconsistent with P2 (Inc).
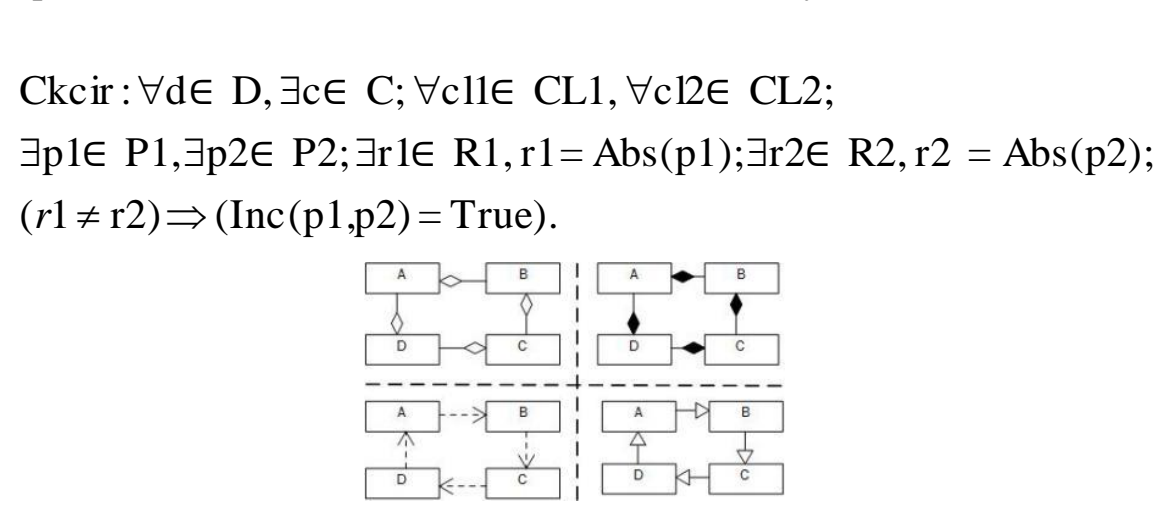

\section{Figure 7. The Circulations}

Figure 7 shows 4 knds of circulation with relationship inconsistency problems. These circulations are aggregation circulation, composition circulation, dependency circulation and generalization circulation. Fo each kind of circulation, there are two relationship results between class $\mathrm{A}$ and class $\mathrm{C}$ and the two relationships are different. Take the aggregation circulation as an example, if we use the relationship abstraction rule (4) to abstract path1:[ $A \times$ (AG) $x D \times(A G) \times C$ ], we get result1:[ $A \times(A G) \times C$ ]. However, if we use rule (4) to abstract path2: $A \times(A G r) \times B \times(A G r) \times C$ ], we get result2: [ $A \times(A G r)$ $x C$ ]. result $1 \neq$ result 2 , so pathl is inconsistent with path2. Figure 8 shows a simple class diagram of the ATM system. The class diagram contains four classes namely Session, Customerm ormation, TransactionRecord and Transaction. According to rule (32): [ (DP) $x$ Class $x(A G)$ equals DP 70 ] given in Figure 8, we abstract the scene as S1:[ Session $x$ (DP) $x$ CustomerInformation $x(A G) \times$ TransactionRecord ]. We get result $r$ l:[ Session $x$ (DP) $x$ TransactionRecord ]. The reliability of $r 1$ is 70. The description of $d 1:[(A G r) x$ Class $x(D P r)]$ is the same as the description of $d 2$ : [ (DP) $x$ Class $x(A G)]$. According to rule (32):[ (DP) x Class $x(A G)$ equals DP 70 ], we abstract the scene S2:[ Session $x$ (AGr) $x$ Transaction $x(D P r) \times$ TransactionRecord ]. We get result $r 2$ : [ Session $x(D P r) x$ TransactionRecord ]. The reliability of $r 2$ is 70 . We conclude that $S 1$ is conflict with $S 2$ since $r l \neq r 2$. The circulation contains inconsistency problem. 


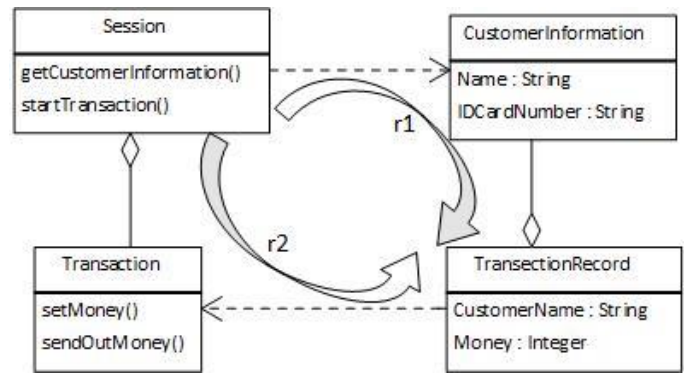

Figure 8. The ATM System Example

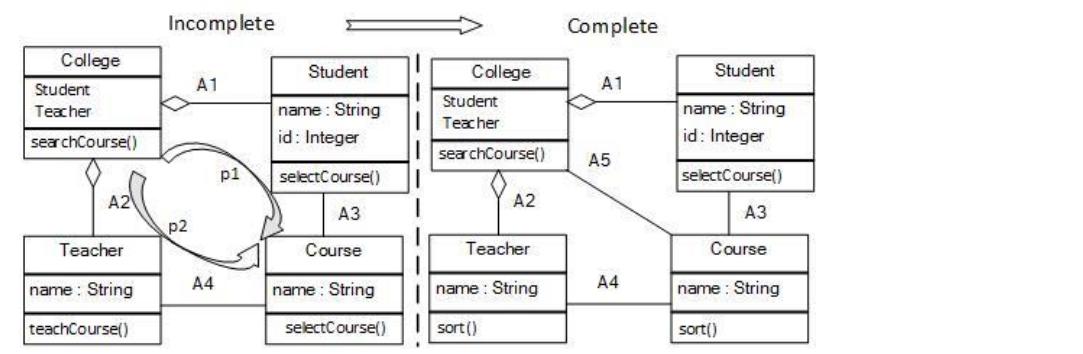

Figure 9. A Relationship Circulation Contains Both Aggregation and Association

2). Completeness of information in circulaton. The completeness problem here mainly refers to the reference integrity. For example, in order to meet the requirements of stakeholders, class A need to get the information in class C. But class A is not directly connected to class C. Class A is conrected to class B and class B is connected to class C. So class A gets the information in crass $\mathrm{C}$ through class B. But sometimes the information is not correct or complete, so the information cannot satisfy all relevant requirements. This kind of problem is the completeness problem. The completeness problem is quite common in circulation. Class diagram in Figure 9 shows relationships between class College, Student, Teacher and Course. Suppose there is a need to find out all the courses in a college. In Figute 9 (left) we find two paths from College to Course. They are p1:[ College $x(A G r(A 1) \times$ Studen $x$ AS(A3)) $x$ Course ] and p2:[ College $x(A G r(A 2)) x$ Teacher $x$ (AS(A4) $x$ Course ]. The courses we get may not only belong to the college, because student in college can choose the courses of other colleges. If we choose $p 2$, the courses we get may not on yelong to the college for there are some visiting professors in the college. Visiting professors may teach some lessons for college students, but they also teach lessons that do not belong to the college. We get a result that no matter through which path $p 1$ or $p 2$, we cannot fully satisfy the requirement. The result is a completeness problem in the circulation. When dealing with this kind of problem, we can add a direct relationship between the two classes. For Figure 9, we can add a direct relationship between class College and Course shown in Figure 9(Right). Using rule (2): [ AS x Class $x$ AO equals AS 100 ], we abstract $p 1$ and get result rl:[ College $x$ AS $x$ Course ]. We abstract $p 2$ and get result $r 2:$ [ College $x$ AS $x$ Course ]. $r 1=r 2$ and the relationship between a college and a course is $A S$ in $r l$ and $r 2$. The type of the direct relationship (A5) between class College and Student is $A S$ according to $r l$ and $r 2$.

3). Relationship redundancy in circulation. Redundancy in circulation means that some relationships are redundancy. Relationship redundancy is also common in a circulation. Relationship redundancy does not cause software errors, but waste the resources such as time and space. We can save resources if we identify and reduce them. Figure 10 shows the relationship redundancy in circulation in Amazon Web Services. Figure 10 illustrates class AwsService, AwsServiceDefinition, AmazonWhishListService and AmazonWhishListServiceDefinition. According to rule (17):[ (GL) x Class $x($ AG) equals 
AG 100 ], we abstract pl: [ AmazonWhishListServiceDefinition $x$ (GL) $x$ AwsServiceDefinition $x$ (AG) $x$ AwsService $]$ and get result rl:[ AmazonWhishListServiceDefinition $x$ (AG) $x$ AwsService ] with reliability 100 . According to rule (27): [ (AG) $x$ Class $x$ (GL) equals $A G 50$ ], we abstract $p 2$ : I AmazonWhishListServiceDefinition $x \quad(A G) \quad x$ AmazonWhishListService $x \quad(G L) \quad x$ AwsService ] and get result r2:[ AmazonWhishListServiceDefinition $x$ (AG) $x$ AwsService ] with reliability 50 . After checking the information completeness of two paths between AmazonWhishListServiceDefinition and AwsService, we find that the information is complete. Because $r l=r 2$, we think that there may be relationship redundancy in the circulation. Because the reliability of $r 1$ is bigger than $r 2$, we suggest that redundancy is existing in $p 2$. We check $p 2$ and speculate that the relationship AG in $p 2$ is redundant and delete AG. Then we check information completeness again. We finally find that there are no problem in the class diagram so we are sure that $A G$ in $p 2$ is a relationship redundancy.
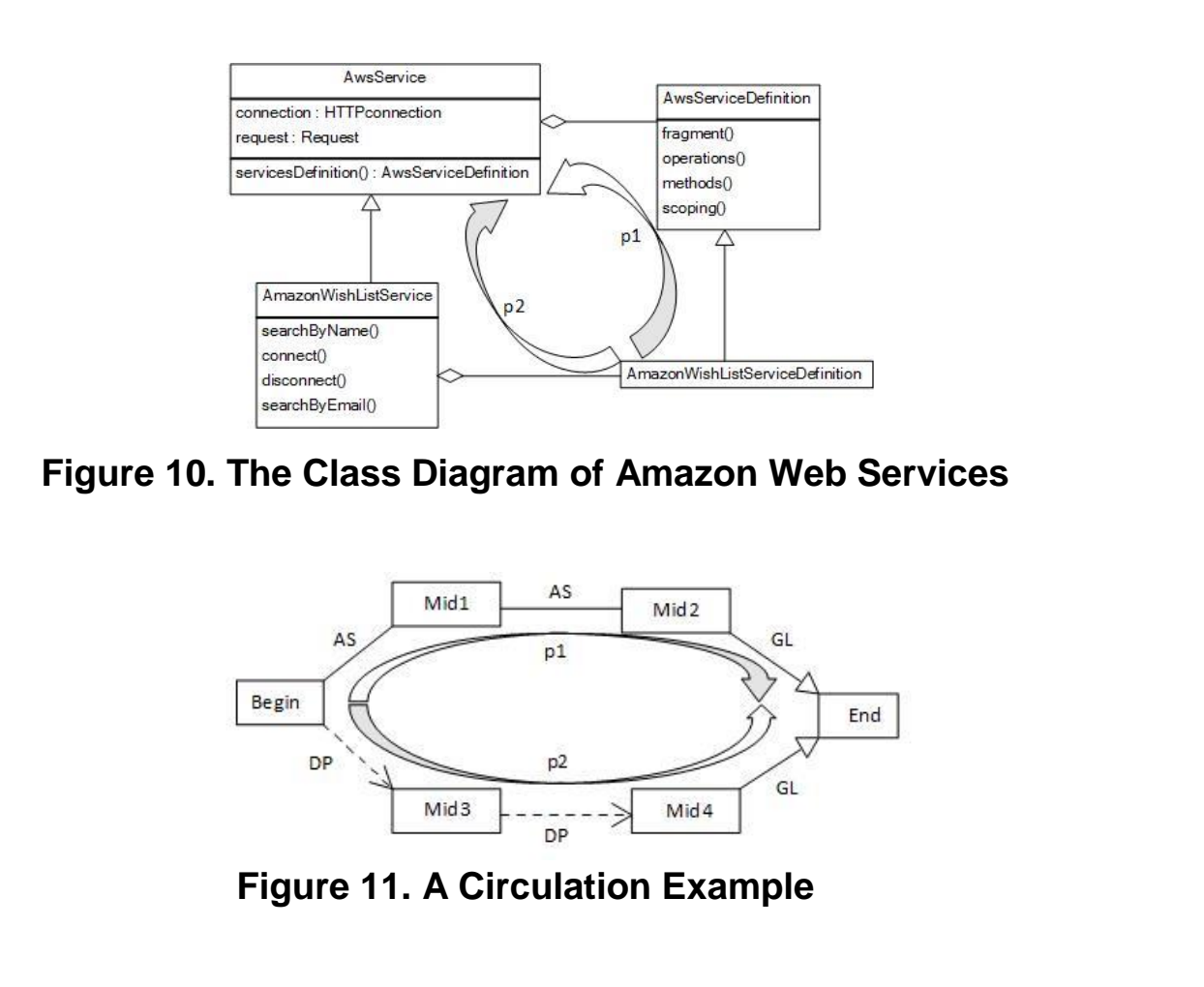

\subsection{Checking Problems in Circulation}

Figure 11 is a simple circulation. In the circulation, between class Begin and class End there ar tyo paths. In order to obtain the relationship between class Begin and class End, we neec to abstract $p 1$ and $p 2$. In order to abstract the two paths and apply the abstraction ules automatically, we use an automaton. Figure 12 shows the method of using an automaton to abstract the two paths. The upper part of Figure 12 shows the automaton for abstracting $p 1$. State $S O$ is the initial state. We describe $S O$ as $S O:[$ (Begin) $x$ (AS) $x$ (Mid1) $x(A S) x(M i d 2) x(G L) x(E n d)]$. Firstly, we use rule (1) to abstract $S 0$, then the state S0 changes to S1. S1 can be described as S1:[ (Begin) $x(A S) x$ (Mid2) $x(G L) x$ (End) ]. Secondly, we use rule (28) to abstract $S 1$ and the state $S 1$ changes to $S 2$. $S 2$ can be described as $S 2:[$ (Begin) $x(A S) x$ (End)]. The reliability of $S 2$ is $100 * 70 / 100=70$. The relationship between class Begin and class End is AS through $p 1$ and the reliability of the relationship is 70 . The lower part of Figure 12 shows the automaton for abstracting $p 2$. $S 3$ is the initial state of $p 2 . S 3$ can be described as S3:[ (Begin) $x(D P) x($ Mid3) $x(D P) x$ (Mid4) $x(G L) x$ (End) ]. Firstly, we use rule (10) to abstract $S 3$ and get $S 4$ :[ (Begin) $x$ 
(DP) $x$ (Mid4) $x(G L) \times(E n d)]$. Secondly, we use rule (34) to abstract $S 4$ and get S5:[ (Begin) $x(D P) x$ (End) ]. The relationship between class Begin and class End is DP through $p 2$. And the reliability of the relationship of $S 5$ is $100 * 50 / 100=50$. After using the automaton to abstract the two paths, The relationship between class Begin and class End in $p 1$ is $\mathrm{AS}$, in $p 2$ is DP. (AS) $\neq(\mathrm{DP})$, so $p 1$ is inconsistent with $p 2$.

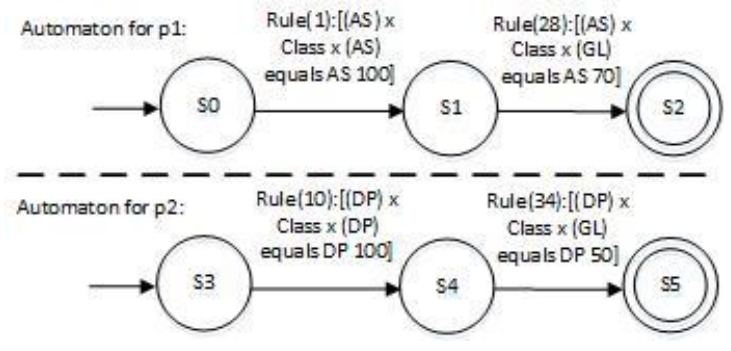

Figure 12. Automata for Abstracting Paths

\section{Inconsistency in a Path}

In the method of using automaton, we apply rules in an iterative way. Through the iterative approach, the final reliability may not be biggest. So we need to find out a new running order to get the biggest reliability. There are two execution orders after we run two rules. Running the first rule firstly or running the second rule firstly leads to two different execution orders. Different execution orders may lead to different results. Figure 13 shows the two execution orders. The 1 ower pant or Figure 13 shows the first execution order. The lower part of Figure 13 executes the rule:[ (RelationshipA) $x$ Class $2 x$ (RelationshipB) equals Relation hipD1 ] firstlyand executes the rule:[ (RelationshipD1) $x$ Class $2 x$ (RelationshipC) equals RelationshipE1 ] secondly. The ultimate relationship of first order between Class Iand Class4 (s RelationshipE1. Reliability of RelationshipE1 is FinalReliabilitya. The upper part of Figure 13 shows the second execution order. The execution apply the rule:[ (RelationshipB) $x$ Class3 $x$ (RelationshipC) equals RelationshipD2 firsty and apply the rule:[ (RelationshipA) $x$ Class $2 \times$ (RelationshipD2) equals Re-lationshipE2 I secondly. The ultimate relationship of second execution order between Class 1 and Class 4 is RelationshipE2. Reliability of RelationshipE2 is FinalReliabilityb. The RelationshipE1 and RelationshipE2 may be different. At the same time the FinalReliability $a$ and FinalReliabilityb may be different too. If RelationshipE1 $\neq$ RelationshipE2 the path contains relationship inconsistency. If FinalReliabilitya $\neq$ FinalReliabilityb, the path contains reliability inconsistency.
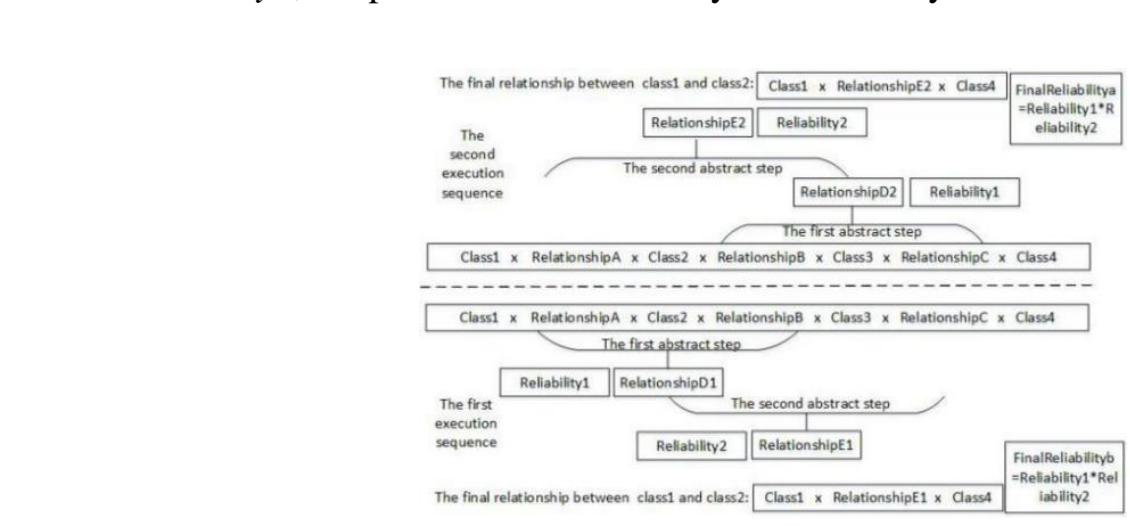

Figure 13. Two Execution Orders 


\subsection{Reliability Inconsistency}

The inconsistency between FinalReliabilitya and FinalReliabilityb belongs to reliability inconsistency. Figure 14 is a scene describes the relationship between class Vegetable, Human, Mammal and Animal. We can describe the scene as S:[ Vegetable $x$ $(A S) x$ Human $x(G L) x$ Mammal $x(G L) x$ Animal $]$. Two relationship abstraction rules in $S$ are rule (28): [ (AS) $x$ class $x(G L)$ equals $A S 70]$ and rule (18): [ $(G L) \times$ class $x(G L)$ equals GL 100 ]. We use the automaton to abstract $S$ iteratively, as the first sequence in Figure 13 shows. Firstly, we apply rule (28) and get the output scene S1:[ Vegetable $x(A S)$ $x$ Mammal $x(G L) x$ Animal ]. Secondly, we still run rule (28) to abstract $S 1$ and get the output scene $S 2:$ [ Vegetable $x(A S) x$ Animal ]. The reliability of $S 2$ is $70 * 70 / 100=49$. However, if we firstly run the rule of which reliability is higher, the results may be different. For the rules in Figure 14, we find that the reliability of rule (18) is 100 and the reliability of rule (28) is 70. The reliability of rule (18) is higher than the reliability of rule (28), so we run rule (18) to abstract $S$ firstly. The second sequence shown in Figure 13. We get the output scene S3: [ Vegetable $x(A S) x$ Human $x(G L) x$ Animal ]. We rum rule (28) to abstract $S 3$ secondly and get the output scene S4:[Vegetable x (AS) x Animal]. Finally the reliability of $S 4$ is $100 * 70 / 100=70$, the value is obviously higher than 49 . Algorithm 1 shows the method of applying the rules of which the reliability is higher firstly and through this method we can get a more reliabe resule.

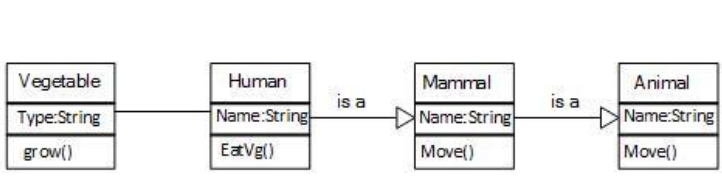

Figure 14. A Sample for the Analysis of Rule Running Sequence
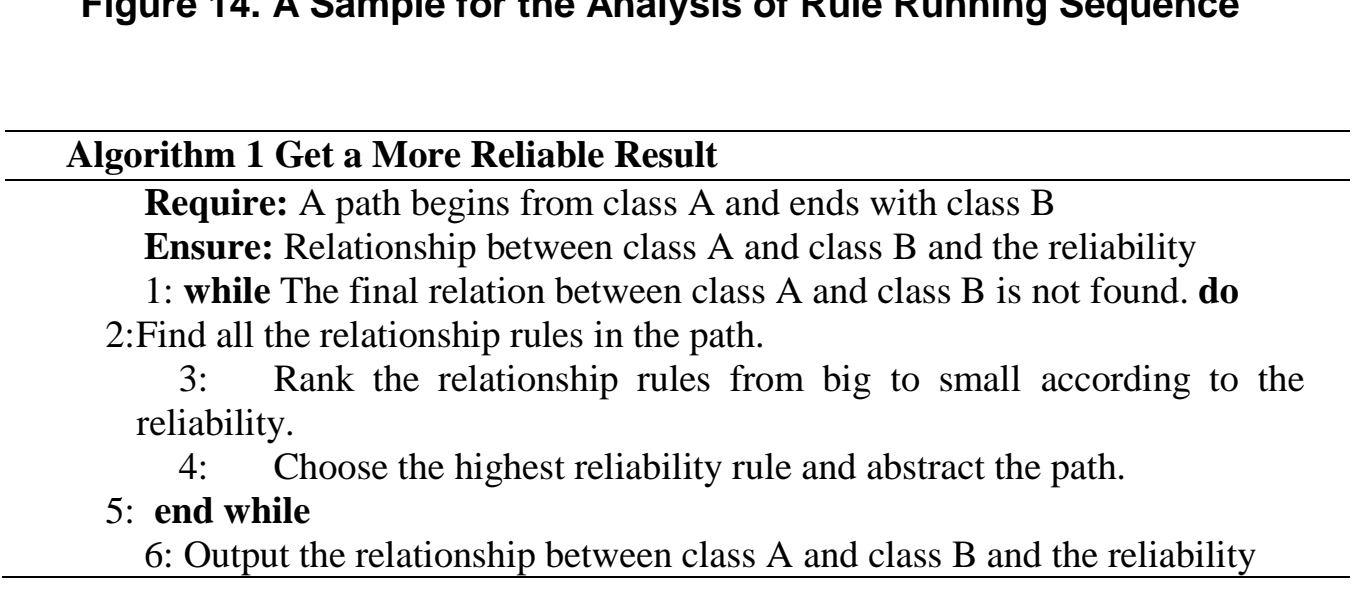

\subsection{Relationship Inconsistency}

The esult we get after abstracting the scene shown in Figure 14 only contains the reliability inconsistency. The consequences of the two relationships are same. The scene does not include relationship inconsistency. However, if $S 2 \neq S 4$, we come to a conclusion that different execution orders have influence on relationship inconsistency. We should consider whether the relationship inconsistency is caused by different execution orders or caused by inconsistency between two paths. If the relationship inconsistency is caused by the different execution orders, we need to eliminate the impact of different execution orders. If the relationship inconsistency is caused by inconsistency between two paths, we draw a conclusion that there are some problems in circulation and we need to modify the information in two paths. In order to eliminate the influence of different execution orders, we need to get all scene types which contain 3 relationships. A scene contains 3 relationships and contains 2 rules. A scene contains two rules and allows two different execution orders. And after analyzing the two execution orders in each scene, we can 
know the impact of different execution orders on circulation checking results. If different execution orders have no impact on scenes which contain two rules, execution orders will not affect the scenes which contain 4 or more relationships. The form of the scene is S: [ Class $x$ (Relationship1) $x$ Class $x$ (Relationship2) $x$ Class $x$ (Relationship3) $x$ Class ]. Relationship1, Relationship2 and Relationship3 are selected from relationship collection $\mathrm{C}=\{\mathrm{AG}, \mathrm{GL}, \mathrm{DP}, \mathrm{AS}, \mathrm{AGr}, \mathrm{DPr}, \mathrm{GLr}\}$. So if we choose one relationship from C and choose three times, we can get all scenes which contains 2 rules, we find that there are 7 relationships in $\mathrm{C}$, so there are $7 * 7 * 7=343$ scenes require analyzing. For each scene type, we compare the two results got from two execution methods. The left of Figure 15 shows the abstracted relationship between row and column. For example, the result (RuleTable[AS][AG]=AS) is built according to rule (2):[ (AS) $x$ Class $x(A G)$ equals $A S$ 100 ]. The right of Figure 15 shows the reliability of each abstraction rule. By searching the two tables we can get the results of two execution orders for 343 scene types.Figure 16 is a

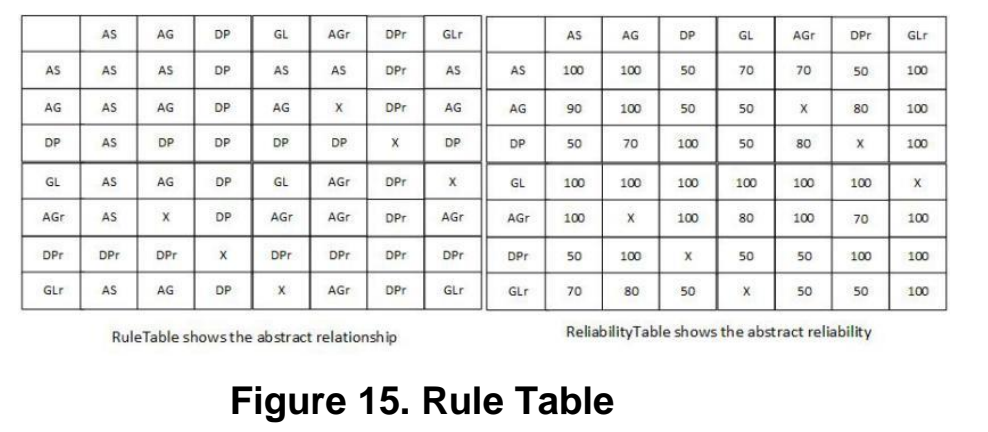

flow chart used to analyze the 343 scene types. Firstly, we select three relationships $\{R 1, R 2, R 3\}$ from the relationship collection $C=\{\mathrm{AG}, \mathrm{GL}, \mathrm{DP}, \mathrm{AS}, \mathrm{AGr}, \mathrm{DPr}, \mathrm{GLr}\}$ in order. And the relationships $Y R I R 2, R 3 \hat{Y}$ can be repetitively selected. And there are 343 kinds of combinations of $\{R 1, R 2, R 3\}$ in order. A $\{R 1, R 2, R 3\}$ order reflect a scene, for a scene, there are two execution orders. We make one order apply rule:[ R1 $x$ Class $x$ $R 2$ equals $R 4$ ] firstly and apply rule:[ $R 4 x$ Class $x R 3$ equals $R a]$ secondly. The application of rules uses the method of checking the rule table and reliability table as shown in Figure 15. We make the another order apply rule:[ R2 $x$ Class $x$ R3 equals $R 5$ ] firstly and apply rule: $[R]$. Class $x R 5$ equals $R b]$ secondly. Finally the two results of the two orders we got are $R a$ and $R b$. If $R a=R b$, for the circulation composed of this scene type, different execulion orders will not result in relationship inconsistency in circulation. If the 343 scene types are not all analyzed, we choose another combination of $\{R 1, R 2$, $R 3\}$ to analyze:

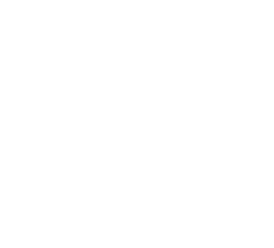




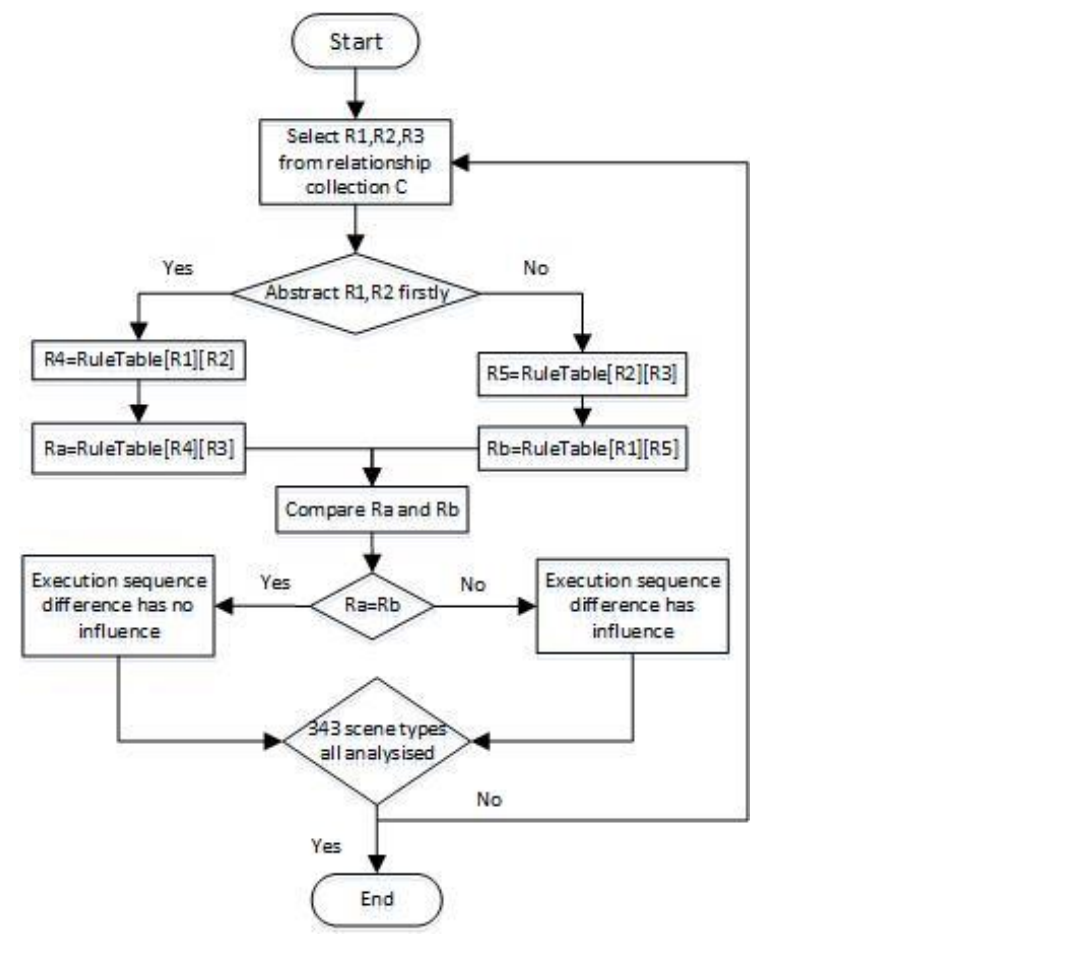

Figure 16. Execution Orders Flowchart

Table 1. Cómparison Results

Table 1. Comparison Results
\begin{tabular}{|l|l|l|}
\hline $\begin{array}{c}\text { Comparison of two } \\
\text { results }\end{array}$ & Percentage \\
\hline Equal & 255 & 0.74 \\
\hline Unequal & 0 & 0.00 \\
\hline Invalid & 88 & 0.26 \\
\hline Total & 343 & 1 \\
\hline
\end{tabular}

Table 1 shown tha result of the experiment. The number of equal type equals to 255 . The number of unequal type-equals to 0 . The number of invalid type equals to 88 . For the equal type and the invalid type we show an example of each type as shown in Figure 17. The top of Figure 17 shows a case that the two results got from two execution orders are equal. The two execution orders are shown in Figure 13. The bottom of Figure 17 shows an invalid type
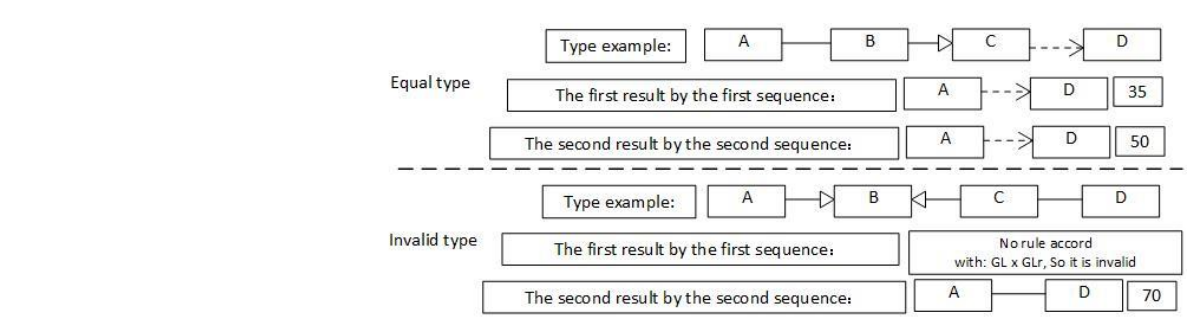

Figure 17. Examples of Different Result Types

After the experiment, we note that there are no cases of unequal type in which the two results of the two execution orders are different. For the invalid type, one execution order is invalid. The bottom of Figure 17 shows a case of invalid type. For this case, we cannot abstract the scene $S:[A \times(G L) \times B \times(G L r) \times C \times(A S) \times D]$ by the first execution order, because there is no rule for the scene: $[A x(G L) \times B \times(G L r) \times C]$. So for invalid type, we 
have only one execution order and can only get a relationship result. So for a circulation contains this kind of scene execution orders will not cause the relationship inconsistency. We conclude that different execution orders will not affect the relationship results of abstraction. In the circulation, If results of abstracting two paths are different, we come to a conclusion that there exists relationship inconsistency between the two paths and the circulation has problems.

\section{Reducing Redundancy and Abstracting a Class Diagram}

\subsection{Checking Redundancy}

If classes in a class diagram have many similarities, redundancy may exist in the class diagram. For example, if two or more classes have same attributes and operations, the same attributes and operations may be redundancy. Figure 18 shows the classes that we used in drawing a graph. The classes contain Ellipse, Polygon, Polyline, PolygonConnector and

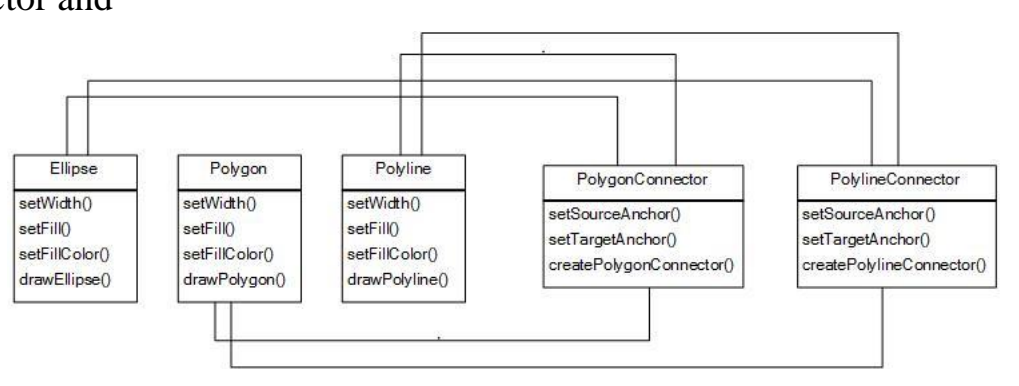

\section{Figure 18. A Simple Craphical Class Diagram}

PolylineConnector. Ellipse, Polygon and Polyline has similar operations. We take their same operations out and put them in a newClass Shape. Then we make class Ellipse, Polygon and Polyline inheri class Shape. We do the same thing with class PolygonConnector and class PolygonConnector. If the limit of a operation is different, we choose the largest number of limit. For example, if the method setWidth() in Ellipse is private, in Polygon is public and $\mathrm{m}$ Polyline is public, the number of the limit which is public equals to 2 , the number of the limit which is private equals to 1 . The largest number of limit equals to 2. So we set the limit of the method setWidth() in Shape public. Through taking same attributes and operations in different classes out, we can reduce redundancy in class diagram. As Figure 18 shows that we needn't code setWidth(), setFill() and setFillCofor() three times in class Ellipse, Polygon and Polyline. The relationship between class Shape and class Connector is based on the relationships between class Ellipse, Polygon, Polyline and class PolygoConnector, polylineConnector.
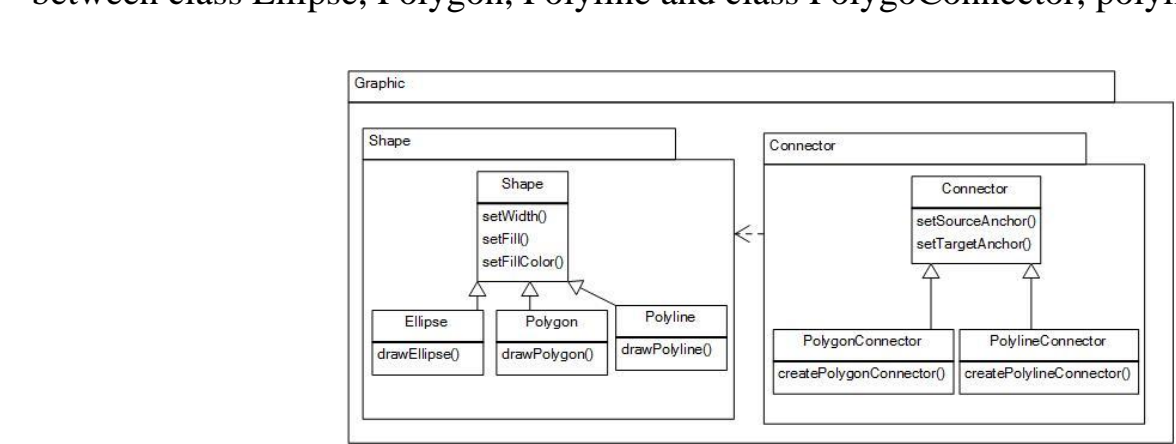

Figure 19. The Class Diagram after Reducing Redundancy 


\subsection{Classifying Classes and Abstracting a Class Diagram}

A package in UML can be viewed as a folder if classes are viewed as files. Package is used to classify classes. In order to get the organizational layer of a software and help us understand the software better, packages are put in place to get the big picture of a software. Figure 20 shows a package diagram abstracted from the class diagram shown in Figure 19. According to the generalization relationships between classes, we can get the scope of a package. But the relationship between packages needs to solve manually.

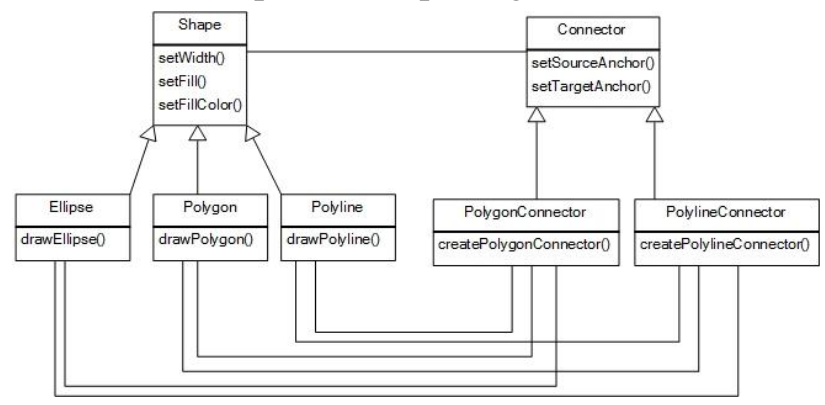

Figure 20. Using a Package to Classify Class Diagrams

\section{Conclusions}

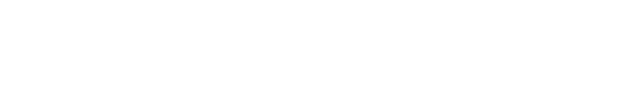

Model abstraction is an extremely importam part in MDE. The abstraction of class diagram takes a big part of model abstraction. The abstraction of a class diagram can help us to understand the software system. We investigate the relationship between classes in a UML class diagram. And we investigate the redundancy of class' attributes and operations. We discover that the circulations in elass diagram often cause problems. We investigate the problems in a clrculation. These problems include relationship inconsistency, completeness problem and relationship redundancy. We investigate how to check these problems and how to address these problems. In the future, we will investigate the cooperation between models. First, we will study the cooperation cases between class diagrams and sequence diagrams. Second, we will investigate the consistency between class diagrams and sequence diagrams.

\section{Acknowledgments}

The authors acknowledge the support of the NSFC of China (No. 61363007 and No. 61462022) and Hainan NSF (No. 20156234), and Postdoctoral Science Foundation of Zhejiang Province of China under Grant No.BSH1502119. * refers to the corresponding author.

\section{References}

[1] D. Brugali, "Model-driven software engineering in robotics: Models are designed to use the relevant things, thereby reducing the complexity and cost in the field of robotics", IEEE Robotics Automation Magazine, vol. 22, no. 3, (2015), pp. 155-166.

[2] Y. Duan, S.-C. Cheung, X. Fu and Y. Gu, "A metamodel based model transformation approach", in Software Engineering Research, Manage- ment and Applications, 2005. Third ACIS International Conference on. IEEE, (2005), pp. 184-191.

[3] A.F. Egyed, "Heterogeneous view integration and its automation", Ph.D. dissertation, University of Southern California, (2000).

[4] R.B. France, D.-K. Kim, S. Ghosh and E. Song, "A uml-based pattern specification technique", Software Engineering, IEEE Transactions on, vol. 30, no. 3, (2004), pp. 193-206.

[5] P. Grunbacher, A.Egyed and N. Medvidovic, "Reconciling software requirements and architectures: the cbsp approach", in Requirements Engineering, 2001. Proceedings. Fifth IEEE International Symposium on. IEEE, (2001), pp. 202-211.

[6] D. Harel and B. Rumpe, "Modeling languages: Syntax, semantics and all that stu", (2000). 
[7] A.G. Kleppe, J. Warmer, W. Bast and M. Explained, "The model driven architecture: practice and promise", (2003).

[8] B.A. Nuseibeh, "A multi-perspective framework for method integra- tion," Ph.D. dissertation, Imperial College, (1994).

[9] M.OMG, "Guide version 1.0. 1", Object Management Group, vol. 62, (2003), pp. 34.

[10] P. J.Puczynski, "Checking consistency between interaction diagrams and state machines in uml models", (2012).

[11] H. Schichl, "Models and the History of Modeling", Springer US, (2004).

[12] R. Soley, D. Frankel, J. Mukerji and E. Castain, "Model driven architecture-the architecture of choice for a changing world", Technical report, OMG, http://www. omg. org, Tech. Rep., (2001).

[13] H. Stachowiak, "Allgemeine modelltheorie", Zitiert auf, (1973),p p. 35.

[14] A. Van.Deursen, E. Visser and J. Warmer, "Model-driven software evolution: A research agenda", Delft University of Technology, Software Engineering Research Group, Tech. Rep., (2007).

\section{Authors}
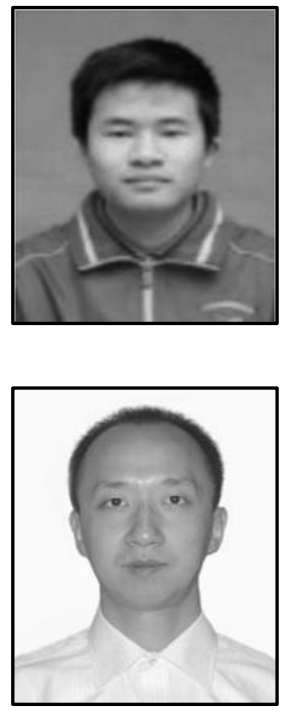

Liang Huang, he is learning Computer Science in Hainan University. His research interests include software engineering service-oriented computing and big data.

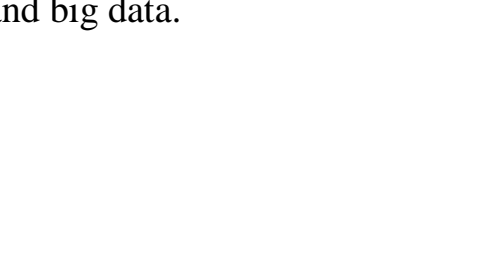

Yucong Duan, he received his $\mathrm{PhD}$ in software engineering from Institute of Softwate, Chinese Academy of Sciences, China in 2006. He is cursently a tull professor and vice director of Computer Science Department in Hainan University, P.R.China. His research interests include software engineering, service computing cloud computing and big data. He is a member of IEEE ACM and CCF (China Computer Federation).

Honghan Gao, he received his $\mathrm{PhD}$ degree in computer application technology from the School of Computer Engineering and Science of Shanghai University, Shanghai, China, in 2012. His research interests include Web service and model checking.

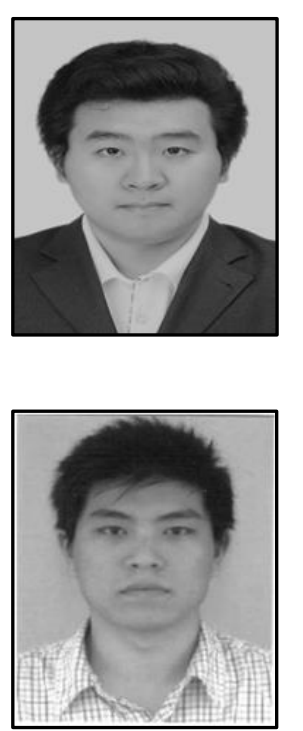

Hui Li, he received his $\mathrm{PhD}$ degree of communication and information system in Department of Electronics and Information Engineering from Harbin Institute of Technology in 2006. He is currently working as a professor in Hainan University. His research interests include advanced wireless networks, space communication and maritime communications. 


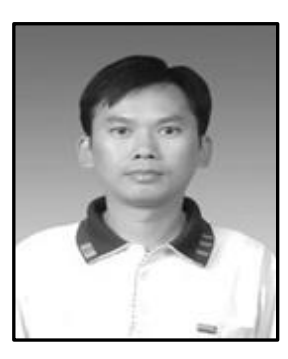

Caimao Li, he received his master's degree in computer application technology from Southeast University in 2004. In 2010, he became an Associate Professor in Department of Computer Science and Technology of Hainan University. His research interests include software engineering, object oriented technology, information security, trusted computing, cloud computing, service computing, web technology and application.

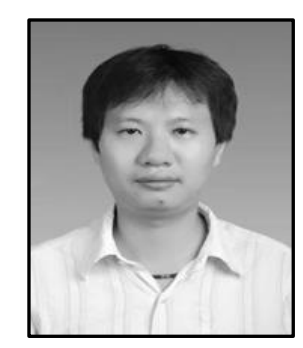

Zhiyang Lin, he received his master's degree in communication and information systems from Hainan University, Haikou, P.R China, in 2008. He is currently teaching in the College of Information Science and Technology in Hainan University. His research interests include mobile communication, image processing and mobile information technology.

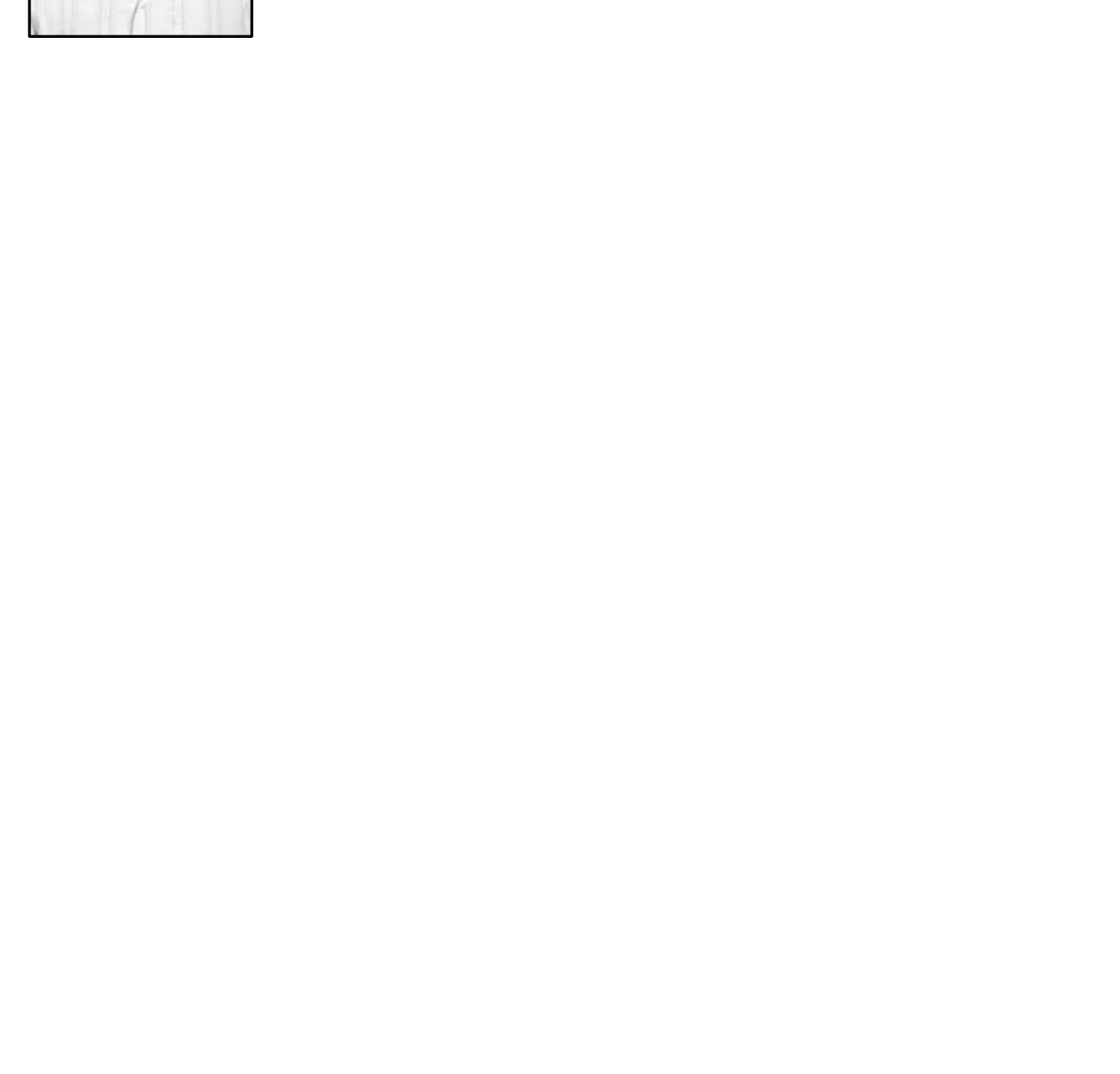

